\title{
Oscillator Phase Noise: A Geometrical Approach
}

\section{Djurhuus, Torsten; Krozer, Viktor; Vidkjær, Jens; Johansen, Tom Keinicke}

\section{Published in:}

I E E E Transactions on Circuits and Systems Part 1: Regular Papers

Link to article, DOI:

10.1109/TCSI.2008.2006211

Publication date:

2009

Document Version

Publisher's PDF, also known as Version of record

Link back to DTU Orbit

\section{Citation (APA):}

Djurhuus, T., Krozer, V., Vidkjær, J., \& Johansen, T. K. (2009). Oscillator Phase Noise: A Geometrical Approach. I E E E Transactions on Circuits and Systems Part 1: Regular Papers, 56(7), 1373-1382. https://doi.org/10.1109/TCSI.2008.2006211

\section{General rights}

Copyright and moral rights for the publications made accessible in the public portal are retained by the authors and/or other copyright owners and it is a condition of accessing publications that users recognise and abide by the legal requirements associated with these rights.

- Users may download and print one copy of any publication from the public portal for the purpose of private study or research.

- You may not further distribute the material or use it for any profit-making activity or commercial gain

- You may freely distribute the URL identifying the publication in the public portal 


\title{
Oscillator Phase Noise: A Geometrical Approach
}

\author{
Torsten Djurhuus, Viktor Krozer, Senior Member, IEEE, Jens Vidkjær, Member, IEEE, and \\ Tom K. Johansen, Member, IEEE
}

\begin{abstract}
We construct a coordinate-independent description of oscillator linear response through a decomposition scheme derived independently of any Floquet theoretic results. Trading matrix algebra for a simpler graphical methodology, the text will present the reader with an opportunity to gain an intuitive understanding of the well-known phase noise macromodel. The topics discussed in this paper include the following: orthogonal decompositions, AM-PM conversion, and nonhyperbolic oscillator noise response.
\end{abstract}

Index Terms-AM-PM, Floquet theory, noise, nonlinear circuits, oscillators, phase macromodel, phase noise.

\section{INTRODUCTION}

$\mathbf{T}$ HE PHASE macromodel is a fast and highly computationally efficient numerical algorithm aimed at phase noise characterization of free-running oscillators, perturbed by noise. The model, which was introduced by Demir et al. in [1] (see also [2] and [3]), follows the earlier work by Kaertner [4], [5] on the subject. The scheme takes outset in a numerically derived periodic steady state with its corresponding monodromy matrix and is hence completely independent of circuit topology and parameters. Using Floquet theory, a noise-forced oscillator phase differential equation is derived and subsequently solved using stochastic integration techniques. The approach described in [1]-[5] is the mathematically correct way of treating the posed problem up to the second order in the noise response.

As could be expected, all this mathematical rigor comes at the price of increased complexity, and the theoretical prerequisites required to fully understand the various derivations leading up to the main results in [1]-[5] are indeed substantial. This could also be the reason why these papers have not received the same level of attention afforded to other more phenomenological contributions on the subject (e.g., [6] and [7]).

This paper seeks to reinterpret this general oscillator noise response model by formulating it in a geometrical context. Avoiding the one-sided algebra-based approach employed in the papers by Demir and Kaertner, our model is based on the simple premise of the following two sets of invariants, which are known to exist for hyperbolic periodic solutions of an autonomous ordinary differential equation (ODE):

1) the limit cycle;

2) the isochrone foliation of the oscillator stable manifold.

Manuscript received February 08, 2008; revised April 29, 2008 and July 28, 2008. First published November 11, 2008; current version published July 01, 2009. This paper was recommended by Associate Editor S. Callegari.

The authors are with the DTU Electrical Engineering Department, Technical University of Denmark, 2800 Kgs. Lyngby, Denmark (e-mail: tdj@elektro.dtu. $\mathrm{dk})$.

Digital Object Identifier 10.1109/TCSI.2008.2006211
The structure following from these basic geometric constructs allows us to partition the state space, leading naturally to the construction of an oscillator coordinate system. The introduction of a proper frame of reference constitutes the premise for the construction of a coordinate-independent model. Using this geometric approach, the oscillator linear response is derived in a very organic and self-contained manner; relying only on what is standard results from differential geometry and linear operator theory while dispensing with the cumbersome Floquet theoretical machinery. The representations used in [1] and [5] will then be derived as a special case, thus furthermore providing an interpretation of the Floquet methodology in a geometrical context.

Having introduced the main topological issues in Section II, Sections III and IV are concerned with deriving a decomposition scheme for vectors and linear maps on the oscillator manifold. The main challenge in this respect comes from the nonorthogonal, or oblique, oscillator coordinate basis. One of the aims of this text will be to investigate this oblique structure of the model in order to better understand its consequences on the oscillator noise response.

Section V contains a short discussion of the inherent coordinate independence of the model which, except for a short note in [5], has not received any significant attention in any of the earlier publications on the subject. We also offer a physical interpretation of the so-called perturbation projection vector $(\mathrm{PPV})$ as a phase differential form. Then, in Section VI, we set out to discuss some interesting topics regarding oscillator noise response inspired by examples taken from the literature, including the validity of an orthogonal decomposition [8], AM-PM conversion [9], [10], and nonhyperbolic oscillator noise response [11]. Finally, Section VII includes a derivation of the asymptotic oscillator phase statistics. In [1], this exercise involved long and somewhat tedious calculations. Following our objective to clarify the formulation, we recapture the main characteristics in a simplified manner.

\section{GEOMETRY OF A Limit CyCle SOlution}

We consider an autonomous ODE

$$
\dot{x}=f(x)
$$

where $(x, f(x))$ defines a vector field on $\mathbb{R}^{n}$, with $x(t): \mathbb{R} \rightarrow$ $\mathbb{R}^{n}$ being the state vector and $f(\cdot): \mathbb{R}^{n} \rightarrow \mathbb{R}^{n}$ supporting all the usual smoothness conditions that are necessary for existence and uniqueness of solutions ${ }^{1}$. The flow, representing the mapping of phase space points along the solution trajectories, is written as $\phi_{\tau}\left(x_{0}\right): \mathbb{R}^{n} \times \mathbb{R} \rightarrow \mathbb{R}^{n}$, where $\tau=t-t_{0}$ denotes a time interval, with $t$ being the absolute time parameter while $t_{0}$ denotes an arbitrary initial time. With this notation, we

\footnotetext{
${ }^{1}$ More specifically, we assume a $C^{\infty}$ vector field where a vector function is of class $C^{k}$ if the partial derivatives, of order $k$ and less, of the vector components all exist and are continuous.
} 
then have $\phi_{0}\left(x_{0}\right)=x_{0}$ and $\phi_{q+s}=\phi_{q} \circ \phi_{s}$ being the usual group property of autonomous flows. With the aforementioned specification of a smooth vector field, the flow becomes a diffeomorphism, i.e., a continuous and smooth map with a likewise continuous and smooth inverse.

In what follows, we assume that (1) contains an attracting and hyperbolic limit cycle ${ }^{2}$, which we shall denote $\gamma$. This compact set is, by definition, mapped invariantly by the nonlinear flow, implying the relation

$$
\phi_{\tau}(\gamma)=\gamma, \quad \text { for all } \tau \text {. }
$$

The oscillator steady-state solution, $x_{s}(t)$, is then a special orbit corresponding to an initial condition in this set, $x_{s}\left(t_{0}\right)=$ $x_{0} \in \gamma, x_{s}\left(t+T_{0}\right)=x_{s}(t)$, with $T_{0}$ being the oscillator period.

There exists a tubular region $W^{s}(\gamma)$, topologically equivalent to $\mathbb{S}^{1} \times \mathbb{R}^{n-1}$ and referred to as the oscillator stable manifold, or simply the oscillator manifold, where, for any $s \in W^{s}(\gamma)$

$$
\lim _{\tau \rightarrow \infty}\left\|\phi_{\tau}(s)-\gamma\right\|=0 .
$$

Considering hyperbolic limit cycles, it is well known that $W^{s}(\gamma)$ can invariantly be foliated into codimension-1 submanifolds, also known as hypersurfaces, diffeomorphic to $\mathbb{R}^{n-1}$ and referred to here as isochrones, with the individual leaves of the foliation crossing the limit cycle transversely [12]-[14]. In this paper, we shall denote these sets as $\rrbracket_{x}$, where the subscript denotes the point of intersection (i.e., $x \in \gamma$ ). The terminology invariantly foliated refers to the fact that the different leaves are permuted by the flow $\phi_{\tau}$

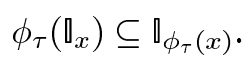

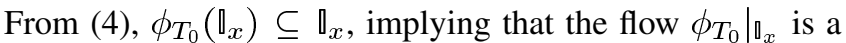
diffeomorphism of $\mathbb{1}_{x}$ into $\mathbb{1}_{x}$, a time- $T_{0}$ return map also known as the Poincaré map.

In the following, we shall also refer to $\rrbracket_{x}$ as a constant phase set, implying that every point contained herein is referenced by the same oscillator phase. This geometric construction then allows for the concept of an oscillator phase to be extended to points off the limit cycle (i.e., to points in $W^{s}(\gamma) \backslash\{\gamma\}$ ). Consider two orbits where one has an initial condition in $\gamma, x \in \gamma$, while the other starts in the corresponding isochrone, $y \in \mathbb{V}_{x}$. From (3) and (4), it follows that

$$
\lim _{\tau \rightarrow \infty}\left\|\phi_{\tau}(x)-\phi_{\tau}(y)\right\|=0 .
$$

As shown in Fig. 1, (5) expresses that points on the same isochrone eventually meet on $\gamma$, with this limit point being the asymptotic phase of points in the set $\mathbb{\square}_{x}$.

The oscillator stable manifold $W^{s}(\gamma)$ is thus naturally divided into two proper submanifolds : 1 ) the 1-D limit cycle $\gamma$ and 2) the $n$-1-dimensional hypersurfaces $\mathbb{\rrbracket}_{x}$. As $\left\{\rrbracket_{x}\right\}_{x \in \gamma}$ divides the state space into constant time/phase "slices" and since time is the parameter of the oscillator integral curve, $x_{s}$, the aforementioned topological constructions introduce the first step toward an oscillator coordinate system on $W^{s}(\gamma)$. The second

\footnotetext{
${ }^{2} \mathrm{~A}$ short discussion of nonhyperbolic oscillator noise response is given in Section VI.D.
}

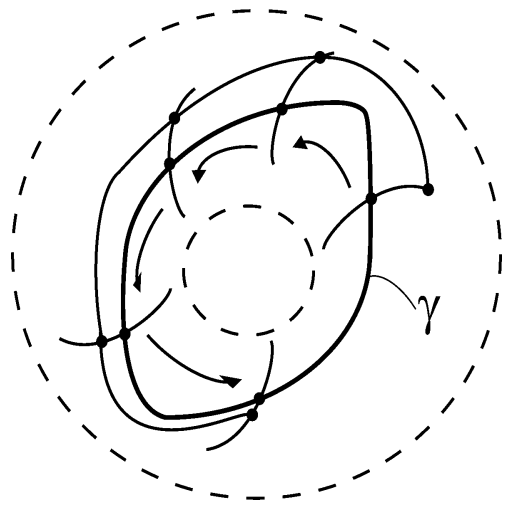

Fig. 1. Assuming a hyperbolic and asymptotically stable limit cycle $\gamma$, there exists an invariant foliation of the oscillator stable manifold $W^{s}(\gamma)$, a tubular region illustrated here with dashed lines. Points on the same leaf of the foliation, known as an isochrone, will converge to the same point on the limit cycle $\gamma$ asymptotically with time, giving rise to the notion of an asymptotic phase. This construction allows for the specification of an oscillator coordinate system.

and final step would then entail the parameterization of the isochrone foliation $\left\{\mathbb{x}_{x}\right\}_{x \in \gamma}$. This construction parallels the way one would create a standard planar Cartesian coordinate system by drawing the $y$-coordinate curves along constant $x$-lines and vice versa. However, unlike the simple Cartesian example, the oscillator coordinate system is generally nonlinear, i.e., the time parameterization on the limit cycle curve is nonlinear.

We are dealing with submanifolds embedded in $\mathbb{R}^{n}$, and their tangent spaces are well defined as affine (translated) subspaces of $\mathbb{R}^{n}$ [15]. In the following, the tangent space of a point $x \in \gamma$ is written as $\mathbb{T}_{x} \mathbb{R}^{n}$. The limit cycle tangent space then becomes ${ }^{3}$ $\mathbb{T}_{x} \mathbb{M}$, while $\mathbb{T}_{x} \rrbracket$ refers to the tangent space of the isochrone leaf at $x \in \gamma$. It then follows that $\mathbb{T}_{x} \mathbb{M}$ and $\mathbb{T}_{x} \rrbracket$ are 1-D and $n-$ 1-dimensional affine subspaces of $\mathbb{R}^{n}$, respectively. Extending the aforesaid definitions of tangent spaces to all points in the limit set $\gamma$ leads to the definition of the tangent bundles $\mathbb{T M}$ and $\mathbb{T} \mathbb{l}$, which are written as ${ }^{4}$

$$
\begin{aligned}
\mathbb{T M} & =\operatorname{span}\left\{u_{1}(t)\right\} \\
\mathbb{T} \mathbb{Q} & =\operatorname{span}\left\{u_{2}(t), u_{3}(t), \ldots, u_{n}(t)\right\}
\end{aligned}
$$

where $u_{i}(t): \mathbb{R} \rightarrow \mathbb{T} \mathbb{R}^{n}$ denotes the tangent bundle vectors also referred to here as modes. From the previous discussion, it should then be clear that, for a hyperbolic oscillator, $\left\{u_{i}(t)\right\}_{i=1}^{n}$ represents a set of linearly independent $C^{\infty} T_{0}$-periodic vectors forming a basis for $\mathbb{T}_{x} \mathbb{R}^{n}$ at each point $x=x_{s}(t) \in \gamma$. This is the natural basis/coordinate basis on the oscillator manifold $W^{s}(\gamma)$, with base space $\gamma$.

The flow on the tangent bundle is governed by the linear response map $D \phi_{\tau}\left(x_{0}\right): \mathbb{T}_{x_{0}} \mathbb{R}^{n} \times \mathbb{R} \rightarrow \mathbb{T}_{\phi_{\tau}\left(x_{0}\right)} \mathbb{R}^{n}$, and as both the limit cycle and the isochrone foliation are invariants of the

\footnotetext{
${ }^{3}$ It is standard notation to refer to the invariant manifold $\gamma$ as $\mathbb{M}$.

${ }^{4} \mathrm{~A}$ more technically correct definition of the tangent bundle would include the specification of a projection $\pi: \mathbb{T} \mathbb{R}^{n} \rightarrow \mathbb{R}^{n}$. Here, we do not specify a projection operator explicitly but instead include the index $t$, referencing the base space as $t \rightarrow x_{s}(t)$

${ }^{5} \mathrm{Here}, D \phi_{\tau}$ is to be understood as the flow resulting from the linearization of the nonlinear flow $\phi_{\tau}\left(x_{0}\right)$, with $x_{0} \in \gamma$. It is a diffeomorphism since $\phi_{\tau}$ is a diffeomorphism.
} 
nonlinear flow $\phi_{\tau}$, it follows that their respective tangent spaces are invariantly mapped by $D \phi_{\tau}$

$$
\begin{aligned}
& D \phi_{\tau}\left(x_{0}\right): \mathbb{T}_{x_{0}} \mathbb{M} \rightarrow \mathbb{T}_{\phi_{\tau}\left(x_{0}\right)} \mathbb{M} \\
& \left.D \phi_{\tau}\left(x_{0}\right): \mathbb{T}_{x_{0}} \mathbb{\rightarrow} \rightarrow \mathbb{T}_{\phi_{\tau}\left(x_{0}\right)}\right)^{\mathbb{l}}
\end{aligned}
$$

In the following, we let the set $\left\{u_{i}\left(t_{0}\right)\right\}_{i=2}^{n}$ in (7) refer to the $n-1$ eigenvectors of the rank $n-1$ return map $\left.D_{T_{0}}\left(x_{0}\right)\right|_{\mathbb{T}_{x_{0}}}$.

State-space points contained in $W^{s}(\gamma)$ must contract, and we have

$$
D \phi_{\tau}\left(x_{0}\right) u_{i}\left(t_{0}\right)=\beta_{i}\left(\tau, x_{0}\right) u_{i}(t)
$$

with $\beta_{i}: \mathbb{R} \times \mathbb{R} \rightarrow \mathbb{R}$ being the contraction function for the $i$ th mode. The bundle $\left\{u_{i}(t)\right\}$ is invariant under the linear response flow, and (10), together with the group property of the linear response $D \phi_{q+s}=D \phi_{q} \circ D \phi_{q}$, which follows from the group property of the nonlinear flow, yields

$$
\begin{aligned}
D \phi_{q+s}\left(x_{0}\right) u_{i}\left(t_{0}\right) & =D \phi_{q}\left(\phi_{s}\left(x_{0}\right)\right) D \phi_{s}\left(x_{0}\right) u_{i}\left(t_{0}\right) \\
& =\beta_{i}\left(q, \phi_{s}\left(x_{0}\right)\right) \beta_{i}\left(s, x_{0}\right) u_{i}(t) \\
& =\beta_{i}\left(q+s, x_{0}\right) u_{i}(t) \Rightarrow \\
\beta_{i}\left(q+s, x_{0}\right) & =\beta_{i}\left(q, \phi_{s}\left(x_{0}\right)\right) \beta_{i}\left(s, x_{0}\right)
\end{aligned}
$$

a group definition for the contraction function. Inserting $q=$ $T_{0}, s=\tau$ in (11)

$$
\beta_{i}\left(T_{0}+\tau, x_{0}\right)=\beta_{i}\left(T_{0}, \phi_{\tau}\left(x_{0}\right)\right) \beta_{i}\left(\tau, x_{0}\right)
$$

then reversing the order in which the intervals are mapped (i.e., $q=\tau, s=T_{0}$ )

$$
\begin{aligned}
\beta_{i}\left(T_{0}+\tau, x_{0}\right) & =\beta_{i}\left(\tau, \phi_{T_{0}}\left(x_{0}\right)\right) \beta_{i}\left(T_{0}, x_{0}\right) \\
& =\beta_{i}\left(\tau, x_{0}\right) \beta_{i}\left(T_{0}, x_{0}\right)
\end{aligned}
$$

and equating (12) and (13)

$$
\beta_{i}\left(T_{0}, \phi_{\tau}\left(x_{0}\right)\right)=\beta_{i}\left(T_{0}, x_{0}\right)=\lambda_{i}
$$

which says that the mode contraction over one period is independent of the initial condition. The constants $\lambda_{i}$, which are known as the characteristic multipliers [15] are uniquely defined by the flow.

The mapping of vectors in the phase bundle TM by the linear response corresponds to trajectories contained entirely in $\gamma$. It follows from (2) that this set can neither contract nor expand under the flow, implying that $D \phi_{\tau}\left(x_{0}\right)$ must display a neutrally stable response

$$
\beta_{1}\left(\tau, x_{0}\right)=1, \quad \text { for all } \tau, x_{0}
$$

and from (14), we then get $\lambda_{1}=1$ while $\left|\lambda_{i}\right|<1$ for $i=$ $2,3, \ldots, n$, which follows from the hyperbolic and asymptotically stable nature of $\gamma$.

Finally, it should be apparent from the previous discussion that

$$
\beta_{i}\left(0, x_{0}\right)=1, \quad \text { for all } i, x_{0}
$$

since $D \phi_{0}$ is, by definition, the identity map.
At this point, we turn to consider the explicit form of the contraction functions $\beta_{i}, i \neq 1$, in (10), and we also say that we choose a representation for the oscillator linear response. Inspecting the group property in (11), it should be clear that $\beta_{i}$ must be some kind of exponential.

The original formulations in [1]-[3] and [5] use the so-called Floquet representation/decomposition that specifies a uniform exponential contraction

$$
\beta_{i}\left(\tau, x_{0}\right)=\exp \left(\mu_{i} \tau\right)
$$

which is easily seen to obey (11)-(16) $\left(\mu_{1}=0\right)$, with the Floquet characteristic exponents $\mu_{i}$ uniquely defined through $\mu_{i}=\ln \left(\lambda_{i}\right) / T_{0}$. As an example of a different class of representations, we consider the planar vector field $f=\left(f_{1}, f_{2}\right)$ and the contraction function

$$
\begin{aligned}
& \beta_{2}\left(\tau, x_{0}\right)=\frac{\left|f\left(x_{0}\right)\right|^{2}}{\left|f\left(\phi_{\tau}\left(x_{0}\right)\right)\right|^{2}} \\
& \times \exp \left(\int_{0}^{\tau} \operatorname{div} f\left(\phi_{\eta}\left(x_{0}\right)\right) \mathrm{d} \eta\right)
\end{aligned}
$$

with $\operatorname{div} f=\partial_{x} f_{1}+\partial_{y} f_{2}$ referring to the divergence of the vector field [16]. Note that, in order for (18) to obey (14), we must have

$$
\frac{1}{T_{0}} \int_{0}^{T_{0}} \operatorname{div} f\left(\phi_{\eta}\left(x_{0}\right)\right) \mathrm{d} \eta=\mu_{2}
$$

and as the divergence is an indicator for oscillator dissipation, we see that the second Floquet exponent represents the timeaverage oscillator dissipation. Although we shall not pursue the topic further here, we note that it should be possible to extend this result to higher dimensional systems, thus allowing for a physical interpretation of all the amplitude Floquet exponents $\left\{\mu_{i}\right\}_{i=2}^{n}$ in terms of oscillator dissipation.

Once a representation has been chosen and the set $\left\{u_{i}\left(t_{0}\right)\right\}_{i=2}^{n}$ has been derived, as discussed previously, the oscillator bundle can be calculated as

$$
u_{i}\left(t_{j+1}\right)=\beta_{i}^{-1}\left(\tau, x_{j}\right) D \phi_{\tau}\left(x_{j}\right) u_{i}\left(t_{j}\right)
$$

where we have divided the cycle interval $\left[t_{0} ; T_{0}+t_{0}\right]$ into equidistant points $\left\{t_{j}\right\}, \tau=t_{j+1}-t_{j}$, with $x_{j}=x_{s}\left(t_{j}\right)$, and used (10) and (11).

\section{Decomposing the Oscillator Tangent Space}

The oscillator coordinate basis introduced in (6) and (7) of the previous section is generally not orthogonal. In fact, as will be discussed in Section V and later in Section VI.C, such an orthogonal decomposition of the tangent bundle is only generated by special oscillator classes.

In the generally nonorthogonal, or oblique, oscillator coordinate system, there exists a preferred decomposition scheme that leads to a coordinate-independent representation of the linear response. However, in order to achieve this structure, we need to introduce the concept of an oscillator cotangent bundle $\mathbb{T}^{*} \mathbb{R}^{n}$, which is spanned not by basis vectors but instead by geometric objects known as one-forms, a special type of tensor. A discussion of tensor analysis as it pertains to oscillator linear response 
is delayed until Section V. Here, we instead turn to the more accessible methods of linear operator theory.

A standard result known as Fredholm's alternative, applied to linear maps, tells us that it is always possible to construct a set of unique linear operators mapping onto the tangent spaces of the manifolds discussed in Section II as long as certain constraints are put on the adjoint operator. We shall use this very general result to construct a set of $n$ oblique projection operators on the oscillator tangent bundle.

The normal space $\mathbb{N}_{x} \mathbb{M}$, at a point $x \in \gamma$, is now defined as the $n$-1-dimensional hyperplane normal to the limit cycle tangent space $\mathbb{T}_{x} \mathbb{M}$. Extending this definition to the whole set $\gamma$, we can hence write the normal bundle $\mathbb{N M}$ as

$$
\mathbb{N M}=\operatorname{span}\left\{v_{2}(t), v_{3}(t), \ldots, v_{n}(t)\right\}
$$

with $v_{i}(t): \mathbb{R} \rightarrow \mathbb{T}^{*} \mathbb{R}^{n}$ being a set of $n$ linearly independent periodic one-forms on the cotangent bundle $6 \mathbb{T}^{*} \mathbb{R}$. From the aforementioned description, it is seen that

$$
u_{1}^{T}(t) v_{i}(t)=0, \quad v_{i}(t) \in \mathbb{N M}
$$

where, in the following, $x^{T}$ refers to the transposition of the vector $x$. It should be noted that, at this point in the analysis, the set $\left\{v_{i}(t)\right\}_{i=1}^{n}$ is not uniquely characterized since a set of forms fulfilling (22) could be chosen in many different ways.

We start by considering the projection onto TM. This operator should have a range that is equal to the limit cycle tangent space and a null response to points in the constant phase manifold $\mathbb{T}$. According to this description, we can define the projection operator $P_{1}(t): \mathbb{T} \mathbb{R}^{n} \rightarrow \mathbb{T} \mathbb{R}^{n}$ as

$$
\text { range }\left\{P_{1}(t)\right\}=\mathbb{T M} \quad \operatorname{null}\left\{P_{1}(t)\right\}=\mathbb{T} \mathbb{0}
$$

From the definitions in (6), (7), and (21), we construct the following two splits of the tangent bundle

$$
\begin{aligned}
& \mathbb{T} \mathbb{R}^{n}=\mathbb{T M} \oplus \mathbb{N M} \\
& \mathbb{T} \mathbb{R}^{n}=\mathbb{V} \mathbb{\oplus} \mathbb{V}_{1}
\end{aligned}
$$

where $\mathbb{V}_{1}$ is the 1-D complement to $\mathbb{\mathbb { Q }}$

$$
\mathbb{V}_{1}=\mathbb{T}^{\perp}=\operatorname{span}\left\{v_{1}(t)\right\}
$$

From (25), it follows that $v_{1}(t)$ must obey

$$
v_{1}^{T}(t) u_{i}(t)=0, \quad u_{i}(t) \in \mathbb{T} \mathbb{\square} .
$$

Note that the set $\left\{v_{1}(t)\right\}$ is now specified, up to a scaling, as the bundle $\mathbb{T} \square$ is uniquely defined (see the discussion in Section II). From the definitions in (24), (25), and (26), we can then write (23)

$$
P_{1}(t)=u_{1}(t) v_{1}^{T}(t) .
$$

${ }^{6}$ See Section V for an explanation of these terms. In order to follow the analysis in this section it suffices to think of one-forms as vectors.
As we consider a projection operator, it follows that $P_{1}(t) \circ$ $P_{1}(t)=P_{1}(t)$, which gives us the relation

$$
\begin{aligned}
u_{1}(t) v_{1}^{T}(t) u_{1}(t) v_{1}^{T}(t) & =u_{1}(t) v_{1}^{T}(t) \Rightarrow \\
v_{1}^{T}(t) u_{1}(t) & =1, \quad \text { for all } t .
\end{aligned}
$$

According to Fredholm's alternative, and the split in (24) and (25), the adjoint operator $P_{1}^{\dagger}(t): \mathbb{T}^{*} \mathbb{R}^{n} \rightarrow \mathbb{T}^{*} \mathbb{R}^{n}$ must then fulfill

$$
\text { range }\left\{P_{1}^{\dagger}(t)\right\}=\mathbb{V}_{1} \quad \text { null }\left\{P_{1}^{\dagger}(t)\right\}=\mathbb{N M}
$$

and we can write ${ }^{7}$

$$
P_{1}^{\dagger}(t)=v_{1}(t) u_{1}^{T}(t)
$$

where the normalization of this operator follows from (29).

It should be apparent from the aforesaid development that we can repeat the process for each of the modes in $\mathbb{T} 0$. This exercise would then lead to a full characterization of the normal bundle $\mathbb{N M}$ defined in (21). We write the split (24) and (25) for the general case as

$$
\begin{aligned}
& \mathbb{T} \mathbb{R}^{n}=\mathbb{U}_{i} \oplus \mathbb{W} \\
& \mathbb{T} \mathbb{R}^{n}=\mathbb{Z} \oplus \mathbb{V}_{i}
\end{aligned}
$$

where $\mathbb{W}=\operatorname{span}\left\{v_{j}(t)\right\}_{j=1, j \neq i}^{n}, \mathbb{U}_{i}=\operatorname{span}\left\{u_{i}(t)\right\}=\mathbb{W}^{\perp}$, $\mathbb{Z}=\operatorname{span}\left\{u_{j}(t)\right\}_{j=1, j \neq i}^{n}$, and $\mathbb{V}_{i}=\operatorname{span}\left\{v_{i}(t)\right\}=\mathbb{Z}^{\perp}$, and we write the set of $n$ oblique projection operators $P_{i}(t)$ : $\mathbb{T} \mathbb{R}^{n} \rightarrow \mathbb{T} \mathbb{R}^{n}$ as

$$
P_{i}(t)=u_{i}(t) v_{i}^{T}(t), \quad i=1,2, \ldots, n
$$

and the corresponding adjoint operators $P_{i}^{\dagger}(t): \mathbb{T}^{*} \mathbb{R}^{n} \rightarrow$ $\mathbb{T}^{*} \mathbb{R}^{n}$ as

$$
P_{i}^{\dagger}(t)=v_{i}(t) u_{i}^{T}(t), \quad i=1,2, \ldots, n .
$$

It follows from the previous discussion (see (27) and (29)) that $\left\{u_{i}\right\}$ and $\left\{v_{i}\right\}$ represent biorthogonal sets

$$
u_{i}^{T}(t) v_{j}(t)=\delta_{i j}, \quad \text { for all } t .
$$

\section{DeCOMposing the Linear Response Flow}

The operator $D \phi_{\tau}\left(x_{0}\right): \mathbb{T} \mathbb{R}^{n} \rightarrow \mathbb{T} \mathbb{R}^{n}$ describing the linear response flow on the oscillator manifold $W_{s}(\gamma)$, with base space $\gamma$, can be divided into the following two separate maps:

1) projection of the initial condition onto proper solution subspaces;

2) mapping the points forward in time.

${ }^{7}$ In the following, we shall only consider 1-D subspaces (modes), which means that $u_{i}^{\dagger}(t) \equiv u_{i}^{T}(t)$ for all $i$. We do not consider 2-D subspaces, which, of course, do occur but which would require the use of complex algebra. The inclusion of the aforementioned manifolds would add nothing to the qualitative understanding of the situation while it would mean a more tedious mathematically complicated notation. The theory described in this paper could very easily be updated to include these 2-D or "complex" results. 
The purpose of the text in the previous section was to derive the projection operation in 1), as given by (34). We then introduce the mapping operator along the $i$ th invariant manifold, which, according to (10), must have the form

$$
M_{i}\left(\tau, x_{0}\right)=\beta_{i}\left(\tau, x_{0}\right) u_{i}(t) u_{i}^{T}\left(t_{0}\right)
$$

where $\beta_{i}$ is the contraction function (see Section II, (12)-(16)) and $\tau=t-t_{0}$ is the time interval. The $i$ th component of the flow $\mathfrak{L}_{i}\left(\tau, x_{0}\right): \mathbb{T} \mathbb{R}^{n} \rightarrow \mathbb{\mathbb { R }} \mathbb{R}^{n}$ is then written as

$$
\begin{aligned}
\mathfrak{L}_{i}\left(\tau, x_{0}\right) & =M_{i}\left(\tau, x_{0}\right) \circ P_{i}\left(t_{0}\right) \\
& =\beta_{i}\left(\tau, x_{0}\right) u_{i}(t) v_{i}^{T}\left(t_{0}\right) .
\end{aligned}
$$

The complete linear response, decomposed according to the oscillator coordinate basis, then becomes

$$
D \phi_{\tau}\left(x_{0}\right)=\sum_{i} \mathfrak{L}_{i}\left(\tau, x_{0}\right)=\sum_{i} \beta_{i}\left(\tau, x_{0}\right) u_{i}(t) v_{i}^{T}\left(t_{0}\right) .
$$

We then turn to consider the adjoint flow, which, according to basic operator theory, is defined through

$$
\left\langle w, \mathfrak{L}_{i}\left(\tau, x_{0}\right) y\right\rangle=\left\langle\mathfrak{L}_{i}^{\dagger}(-\tau, x) w, y\right\rangle
$$

where $y$ is a vector in the tangent bundle at the point $x_{s}\left(t_{0}\right)=$ $x_{0}, y \in \mathbb{T}_{x_{0}} \mathbb{R}^{n}, w$ is a one-form in the cotangent bundle at the point $x_{s}(t)=x, w \in \mathbb{T}_{x}^{*} \mathbb{R}^{n}$, and $\langle\cdot\rangle$ denotes the scalar product. Decomposing the vector $y$ and one-form $w$ according to the scheme developed in Section III, we get $w=\sum_{i} a_{i} v_{i}(t), y=$ $\sum_{i} b_{i} u_{i}\left(t_{0}\right)$, with $a_{i}=u_{i}^{T}(t) w$ and $b_{i}=v_{i}^{T}\left(t_{0}\right) y$. Inserting these into (40) and using the identity $\beta_{i}\left(\tau, x_{0}\right)=1 / \beta_{i}(-\tau, x)$, which follows from (11) and (16) $\left(x=\phi_{\tau}\left(x_{0}\right)\right)$, we find the following expression for the $i$ 'th component of the adjoint flow $\mathfrak{L}_{i}^{\dagger}\left(\tau, x_{0}\right): \mathbb{T}^{*} \mathbb{R}^{n} \rightarrow \mathbb{T}^{*} \mathbb{R}^{n}$

$$
\mathfrak{L}_{i}^{\dagger}(\tau, x)=\beta_{i}^{-1}(\tau, x) v_{i}\left(t_{0}\right) u_{i}^{T}(t)
$$

leading to the following decomposition of the adjoint linear response operator:

$$
D \phi_{\tau}^{\dagger}(x)=\sum_{i} \mathfrak{L}_{i}^{\dagger}(\tau, x)=\sum_{i} \beta_{i}^{-1}(\tau, x) v_{i}\left(t_{0}\right) u_{i}^{T}(t) .
$$

Holding $t$ fixed while interpreting $t_{0}$ as the new time parameter, the adjoint flow can be divided into two maps as follows:

1) projecting the solution onto the corresponding initial condition manifold;

2) bringing the points backward in time.

The monodromy matrix is defined as the time- $T_{0}$ return map for the linearized flow. Using the decomposition in (39), this can be written as

$$
\begin{aligned}
\Phi & =D \phi_{T_{0}}\left(x_{0}\right)=\sum_{i} \beta_{i}\left(T_{0}, x_{0}\right) u_{i}\left(t_{0}+T_{0}\right) v_{i}^{T}\left(t_{0}\right) \\
& =\sum_{i} \beta_{i}\left(T_{0}, x_{0}\right) u_{i}\left(t_{0}\right) v_{i}^{T}\left(t_{0}\right)=\sum_{i} \lambda_{i} P_{i}\left(t_{0}\right)
\end{aligned}
$$

where $\lambda_{i}$ was defined in (14). Equation (43) illustrates how, in the course of a single cycle, the linear response flow of the mode $u_{i}(t)$ is contracted by a factor $0<\left|\lambda_{i}\right| \leq 1$.

\section{COORDINATE-INDEPENDENT REPRESENTATION}

Using standard definitions from differential geometry, it follows that the set $\left\{v_{i}(t)\right\}_{i=1}^{n}$, for a given $t$, represents a coordinate basis for the dual tangent space $\mathbb{T}_{x}^{*} \mathbb{R}^{n}, x \in \gamma$, the so-called dual basis, and, hence, that $\operatorname{span}\left\{v_{i}(t)\right\}_{i=1}^{n}=\mathbb{T}^{*} \mathbb{R}^{n}$, with $\mathbb{T}^{*} \mathbb{R}^{n}$ being the so-called co-tangent bundle. This in turn leads to the definition of a co-tangent phase bundle $\mathbb{T}^{*} \mathbf{M}=\operatorname{span}\left\{v_{1}(t)\right\}$, and a cotangent constant phase bundle $\mathbb{T}^{*} \mathbb{N}=\operatorname{span}\left\{v_{i}(t)\right\}_{i=2}^{n}$. The vectors in $\mathbb{T} \mathbb{R}^{n}$, spanned by the basis $\left\{u_{i}(t)\right\}_{i=1}^{n}$, and the one-forms in $\mathbb{T}^{*} \mathbb{R}^{n}$, spanned by the dual basis $\left\{v_{i}(t)\right\}_{i=1}^{n}$, are different geometric objects in the sense that they transform in an opposite manner ${ }^{8}$. The vectors in $\mathbb{T} \mathbb{R}^{n}$ can then be thought of as geometric objects that "point" in a certain direction, independently of the coordinate basis, while one-forms in $\mathbb{T}^{*} \mathbb{R}^{n}$ are functionals on the vector space $\mathbb{T} \mathbb{R}^{n}$. With the aforementioned definitions, the projection operators in (34) are $(1,1)$ tensors (i.e., linear operators) for a fixed $t$, where the term "tensor" is used to refer to any coordinate-independent geometric object".

In previous papers dealing with the so-called phase macromodel, some authors refer to the set $\left\{v_{1}(t)\right\}$ as the PPV. However, this name is actually somewhat misleading since it suggests that these objects should transform as vectors, which they do not, and since it completely misses the physical interpretation that follows from the aforesaid text. The geometrically correct picture of the PPV as a one-form, a phase differential form, and, hence, the covariant version of the phase function ${ }^{10} \mathrm{gra}$ dient vector $\nabla \alpha$ on $W^{s}(\gamma)$ is shown in Fig. 2.

Using standard topological considerations, we have created a coordinate description on the oscillator manifold $W^{s}(\gamma)$, and as long as a coordinate transformation is nonsingular, it is perfectly legal to change the coordinate basis. All geometric objects (i.e., scalars, vectors, one-forms, and higher order tensors) will maintain their original interpretation in the new frame (see Footnote 9).

In [5], Kaertner also noted this coordinate independence for the Floquet representation. He then proceeded to give an example of a model that did not include this property. In an earlier paper [4], the phase projection operator was defined as

$$
P=\dot{x}_{s} \dot{x}_{s}^{T} /\left\|\dot{x}_{s}\right\|^{2}
$$

${ }^{8} \mathrm{We}$ also say that vectors are contravariant since their components transform oppositely compared to the basis, while one-forms are covariant because their components transform in the same manner as the basis. Note that standard notation stipulates that contravariant objects have raised indexes (i.e., $v^{j}$ ). Here, we do follow these index rules since we want to stay close to notation from [1].

${ }^{9}$ The expressions in (34) are coordinate independent since $w^{T} P_{i} y$ with $w \in$ $\mathbb{T} * \mathbb{R}^{n}$ and $y \in \mathbb{T} \mathbb{R}^{n}$ is a true scalar i.e., a number independent of basis. Considering the linear transformation $x^{\prime}=A x$, with $A$ being a nonsingular $n \times n$ matrix, the vector transforms as $y^{\prime}=A y$ and the one-form as $w^{\prime T}=w^{T} A^{-1}$, while from (34), we have $P_{i}^{\prime}=A P_{i} A^{-1}$, and the coordinate independence follows directly. The aforementioned results can be extended to nonlinear coordinate transformations.

${ }^{10} \mathrm{Here}$, we follow [1] and label the oscillator asymptotic phase $\alpha$ (see also the discussion in Section VII). 
which is easily seen not to be a tensor, except for special cases; in fact, referring to Footnote 9, (44) only transforms as a tensor if the transformation has the special symmetry $A^{T}=A^{-1}$. The expression in (44) hence only constitutes a phase projection operator, in the coordinate-independent sense of the word, if the oscillator supports an orthogonal coordinate basis.

\section{APPLICATIONS}

In this section, we apply the developed methodology to a collection of simple planar oscillators from the literature. This exercise will serve to illustrate some of the characteristic issues in the linear response analysis of more complex systems. The geometrical approach will be seen to lead to a simplified, easy-to-understand, and almost algebra-free analysis.

\section{A. Coram's Nonorthogonal Oscillator}

In [8], Coram investigates the topic of orthogonal decompositions of the oscillator linear response by considering the planar ODE system

$$
\begin{aligned}
& \dot{r}=r(1-r) \\
& \dot{\phi}=1+r
\end{aligned}
$$

which includes the asymptotically stable hyperbolic limit cycle $(r, \phi)=\left(1,2 t+\phi_{0}\right)$. Using standard Floquet analysis for his investigation of (45) and (46), the author achieves in illustrating that the assumption of an orthogonal decomposition, used in certain earlier publications on the subject, is not always valid. The main points of this analysis can be attained almost effortlessly and, with only a minimum use of algebra, by applying the geometric tools developed in this paper.

The vector field in (45) and (46) is rotationally symmetric, and any invariant foliation must preserve this symmetry. Then, we have

$$
\llbracket(\phi, r)=\phi-f(r)
$$

where $f: \mathbb{R} \rightarrow \mathbb{R}$ is a scalar function to be determined. Differentiating the aforementioned expression with regard to time, we get

$$
\frac{\mathrm{d}}{\mathrm{d} t}(\mathbb{\square}(\phi, r))=\frac{\mathrm{d} \phi}{\mathrm{d} t}-\frac{\mathrm{d} f}{\mathrm{~d} r} \frac{\mathrm{d} r}{\mathrm{~d} t} \Leftrightarrow \frac{\mathrm{d} f}{\mathrm{~d} r}=-\frac{\frac{\mathrm{d} \mathbb{d} t}{\mathrm{~d} t}-\frac{\mathrm{d} \phi}{\mathrm{d} t}}{\frac{\mathrm{d} r}{\mathrm{~d} t}} .
$$

Since all points on the isochrone move with the same frequency as the limit cycle points, we have $\dot{\mathbb{1}}=2$. Using this property, together with (45) and (46), we find the following solution to $(48)$ :

$$
\frac{\mathrm{d} f}{\mathrm{~d} r}=-\frac{2-(1+r)}{r(1-r)}=-\frac{1}{r} \Rightarrow f(r)=-\ln (r)+C
$$

where $C$ is an integration constant. The function $f$ has a singularity at $r=0$. However, since we only assume that the invariant foliation exists in a tubular region around $r=1$ (see the discussion in Section II), this is not a problem. Enforcing ! $=\phi$ on the limit cycle $r=1$ yields $C=0$. From (47), the isochrone foliation for the system in (45) and (46) is then written as

$$
\mathbb{\square}(\phi, r)=\phi+\ln (r)
$$

The amplitude-mode vector $u_{2}$ is now found as the tangent vector of the parameterized curve $(\phi(r), r)=(\Theta-\ln (r), r)$

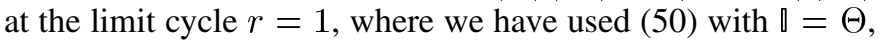
an arbitrary constant phase, to express $\phi$ as a function of $r$. We then find

$$
u_{2}=\mathrm{d}(\Theta-\ln (r), r) /\left.\mathrm{d} r\right|_{r=1}=-\hat{\phi}+\hat{r}
$$

where $(\hat{r}, \hat{\phi})$ is the usual polar basis. Since we have $u_{1}=\hat{\phi}$, the linear response decomposition of the system in (45) and (46) is seen to be nonorthogonal.

\section{B. AM-PM Noise Conversion (I): The Oscillator $Q$}

In this section, we reconsider (45) and (46) from the previous section in a slightly modified form and with noise forcing

$$
\begin{aligned}
\dot{r} & =\alpha r(1-r)+\xi_{1} \\
\dot{\phi} & =2 Q+\beta r+\xi_{2}
\end{aligned}
$$

where differentiations are now performed with regard to the slow time $\tau=t \omega_{3 \mathrm{~dB}}=t \omega_{0} / 2 Q, \omega_{0}$ is the resonator natural frequency, $Q$ is the quality factor of the resonator, $\alpha>0$ determines the stiffness of the amplitude regulation, and $\beta \in \mathbb{R}$ denotes the amplitude dependence of the frequency, which is referred to here as the frequency modulation index. The two noise sources $\left(\xi_{1}, \xi_{2}\right)$ are uncorrelated Gaussian white noise sources with power $\left\langle\xi_{i}^{2}\right\rangle=2 N_{0}$, with $N_{0}=4 k T$ being the single-sided available noise power in a $1-\mathrm{Hz}$ bandwidth, and the extra factor two follows because we consider narrow-band noise sources [17]. We then interpret (52) and (53) as the noise-forced averaged state equations of a high- $Q$ harmonic oscillator with resonator bandwidth $\omega_{3 \mathrm{~dB}}$ (see, e.g., [9] and [10]).

Repeating the calculations that lead to (50) in the previous section, we find the isochrone foliation for (52) and (53)

$$
\llbracket(\phi, r)=\phi+(\beta / \alpha) \ln (r)=\phi+\tan (\psi) \ln (r)
$$

and the isochrone tangent bundle is then given as

$$
u_{2}=-(\beta / \alpha) \hat{\phi}+\hat{r}=-\tan (\psi) \hat{\phi}+\hat{r}
$$

where $\psi$ in the aforementioned equations represents the angle between the isochrone and radial, which is referred to here as the isochrone opening angle ${ }^{11}$. Equation (54) is shown in Fig. 3 for three different values of $\alpha$.

\footnotetext{
${ }^{11} \cos (\psi)=\left(\hat{r} \cdot u_{2}\right) /\left(\left|\hat{r} \| u_{2}\right|\right)=(1) /\left(\sqrt{((\beta) /(\alpha))^{2}+1}\right) \Rightarrow \tan (\psi)=$ $(\beta) /(\alpha)$
} 


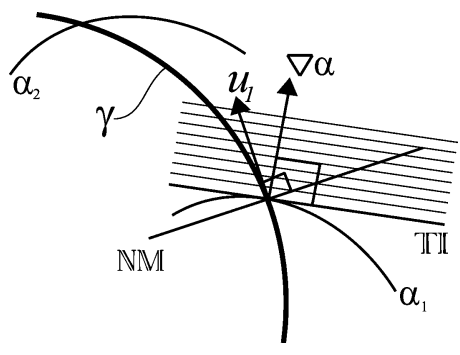

Fig. 2. One-forms $v_{1}$ span the cotangent phase bundle $\mathbb{T} * \mathbb{M}$ and are interpreted physically as phase differential forms $\mathrm{d} \alpha$ (see Footnote 10). We can visualize such a form as a series of $n-1$-dimensional parallel hyperplanes generated by the constant phase tangent space $\mathbb{T} \mathbb{D}$. The contraction of $\mathrm{d} \alpha$ with vectors in $\mathbb{T} \mathbb{R}^{n}$ is then determined by the number of surfaces that these vectors pierce. The contravariant version of $\mathrm{d} \alpha$ is the phase function gradient vector $\nabla \alpha$ that points in a direction that is normal to the constant phase level sets i.e., the direction of maximal phase change. There exists an equivalent physical interpretation of the forms $v_{2}-v_{n}$ living in the normal space $\mathbb{N M}$, where, now, an oscillator energy function takes the place of the phase function $\alpha$.

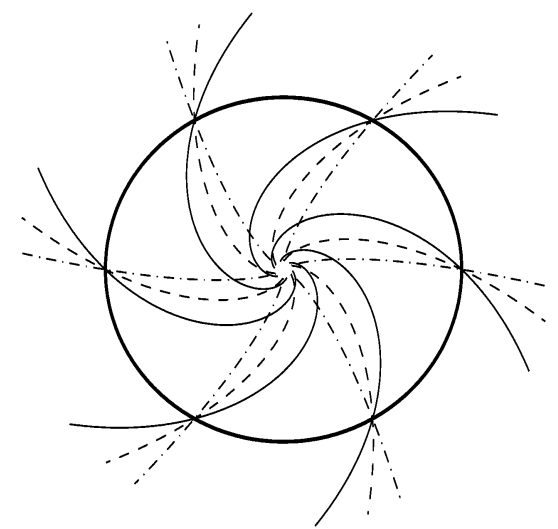

Fig. 3. Limit cycle and isochrone foliation of the oscillator in (52) and (53)

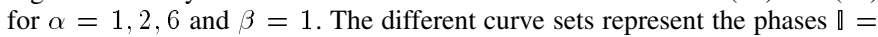
$0, \pi / 6, \pi / 3, \pi, 2 \pi / 3,5 \pi / 6$. Solid line $[-]: \alpha=1$. Broken line [--]: $\alpha=2$. Dashed-dot $[--]: \alpha=6$. Note that (solid line) $\beta=1, \alpha=1$ also represents the isochrone foliation of (45) and (46), as calculated in (50).

The standard algorithm for noise analysis of an ODE like (52) and (53), laid out in [1], specifies the derivation of the so-called phase PPV $v_{1}$, which actually is not a vector (see the discussion in Section V) and which is then used to isolate the phase stochastic differential equation (SDE). Finally, this equation is solved using stochastic integration. We will have more to say about this algorithm in Section VII, where we deal with the derivation of the asymptotic phase statistics. However, here, we want to illustrate an alternative approach.

We now introduce the coordinate transformation $\phi \rightarrow \phi+$ $\tan (\psi) r$, which leads to the new state equations in the transformed frame

$$
\begin{aligned}
& \dot{r}=\alpha r(1-r)+\xi_{1} \\
& \dot{\phi}=2 Q+\beta r+\beta r(1-r)+\xi_{2}+\tan (\psi) \xi_{1}
\end{aligned}
$$

with an isochrone foliation being shown in Fig. 4. From this figure, it should be clear that we have created an orthogonal decomposition for the linear response (i.e., $\psi=0^{\circ}$ ). In this orthogonal system, the amplitude and phase are decoupled, and we then do not need to define a PPV but can instead directly integrate (57) in linearized form. As the two noise sources on the right-hand side of (57) are uncorrelated, this leads to the

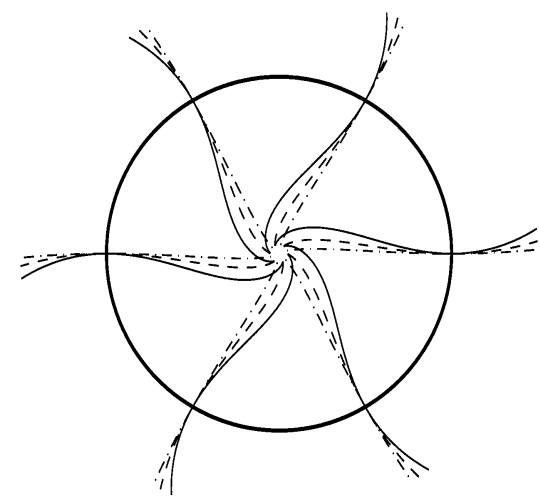

Fig. 4. Isochrone foliation for (52) and (53) in the transformed frame $\phi \rightarrow$ $\phi+(\beta / \alpha) r$ becomes $\mathbb{D}(\phi, r)=\phi+(\beta / \alpha) \ln (r)+(\beta / \alpha)(1-r)$, where the parameter sets corresponding to the different plot symbols are explained in the caption of Fig. 3.

definition of a phase diffusion constant $D_{\phi \phi}$, which is referred to as $c$ in [1] (see also Section VII) ${ }^{12}$

$$
\begin{aligned}
D_{\phi \phi} & =\lim _{t \rightarrow \infty} \frac{\left\langle(\phi(t)-\phi(0))^{2}\right\rangle}{2 t} \\
& =\frac{1}{2}\left(\frac{\omega_{0}}{2 Q}\right)^{2}\left\{1+\tan ^{2}(\psi)\right\} \frac{N_{0}}{P_{0}}
\end{aligned}
$$

where $\phi$ now refers to the linear response variable with renormalized time, $P_{0}=\hat{r}^{2} / 2$ is the oscillator power, $\hat{r}$ is the steadystate amplitude that is one. Note that $P_{0}$ enters the denominator of (58) since the correct angle coordinate is $r \phi$ and not simply $\phi$. The standard expression for the single-sideband noise spectrum of a harmonic feedback oscillator, known as the Leeson model [19], is $\mathfrak{L}\left(\omega_{m}\right)=\left(\omega_{0} / Q \omega_{m}\right)^{2}\left(N_{0} / 2\right) / P_{0}$, where $\omega_{m}$ is the offset from $\omega_{0}$. With the notation from [1], this becomes $\mathfrak{L}\left(\omega_{m}\right)=\left(\omega_{0} / \omega_{m}\right)^{2} D_{\phi \phi}$, and relating the two expressions, we then get $Q=\sqrt{N_{0} / P_{0} 2 D_{\phi \phi}}$. Using (58) in this expression, we can define an oscillator $Q$-factor $Q_{\mathrm{osc}}$ as

$$
Q_{\mathrm{osc}}=\frac{Q}{\sqrt{1+\tan ^{2}(\psi)}}=Q \cos (\psi) .
$$

From (58) and (59), we hence see that AM-PM noise conversion is proportional to the tangent of the isochrone opening angle, while the oscillator $Q$-factor is proportional to the cosine of the same angle. The expression in (59) can be seen as a nonlinear extension of Razavi's oscillator $Q$ [20], which was derived using a linear feedback model.

From the expression $\tan (\psi)=\beta / \alpha$ (see Footnote 11), it follows that the amplitude regulation $\alpha$ and the frequency modulation index $\beta$ are dual parameters in the sense that $\alpha(\uparrow \downarrow), \beta(\downarrow \uparrow$ )$\Rightarrow \psi(\downarrow \uparrow)$, as can also be observed from Fig. 3. It then follows from (59) that one can increase the oscillator $Q$ by (either) decreasing the modulation index $\beta$ by, e.g., making the phase characteristic of the resonator as flat as possible at the oscillation frequency and/or by increasing the stiffness of the amplitude regulation.

As mentioned earlier, simple expressions like (52) and (53) appear as averaged models of more complex harmonic oscillators. An example of this was given in [9], which considered

\footnotetext{
${ }^{12}$ Here, we use that $[18]\left\langle\left(\phi(t)-\phi\left(t_{0}\right)\right)^{2}\right\rangle=\left\langle\left(\int_{0}^{t} \xi_{2}(\eta) \mathrm{d} \eta+\right.\right.$ $\left.\left.\tan (\psi) \int_{0}^{t} \xi_{1}(\eta) \mathrm{d} \eta\right)^{2}\right\rangle=2 N_{0} t\left[1+\tan ^{2}(\psi)\right]$, where we have set $t_{0}=0$.
} 
noise analysis of quadrature oscillators. Expressions, which are similar in form to (58) and (59), also appear in (23) and (29) of that paper, thus illustrating the generality of the aforementioned expressions.

\section{AM-PM Noise Conversion (II): The Nonsymmetric Case}

The discussion in the previous sections concerning a possible orthogonal decomposition appears to be somewhat misplaced, or artificial, when entering the domain of "real-life" oscillators where the limit cycles are not rotationally symmetric. Here, we consider the simple planar ODE

$$
\begin{aligned}
& \dot{x}=f_{1}(x, y)=-y \\
& \dot{y}=f_{2}(x, y)=-\mu\left(1-x^{2}\right) y+x
\end{aligned}
$$

known as the van der Pol oscillator, where $\mu \geq 0$ is a positive parameter that is proportional to the dissipation. For zero dissipation $(\mu=0)(60)$ and (61) become a so-called Hamiltonian oscillator. In the following, we shall use the notation $f=\left(f_{1}, f_{2}\right)$ to refer to (60) and (61), while $f^{\perp}=\left(f_{2},-f_{1}\right)$ denotes the orthogonal field. In order for $f^{\perp}$ to generate an invariant foliation for (60) and (61), its Lie derivative with this vector field should be proportional to $f^{\perp}$

$$
\mathfrak{L}_{f} f^{\perp}=\left[f, f^{\perp}\right]=\zeta(x, y) f^{\perp}
$$

where $\zeta$ is a function that depends on the representation (see Section II) and $\left[f, f^{\perp}\right]$ is the so-called Lie bracket. Using the notation $\left[f, f^{\perp}\right]=\left(w_{1}, w_{2}\right)$, a simple calculation gives ${ }^{13}$

$$
\begin{aligned}
& w_{1}(x, y)=-\mu x\left(2 y^{2}+\left(1-x^{2}\right)\right)+O\left(\mu^{2}\right) \\
& w_{2}(x, y)=-\mu y\left(2 x^{2}-\left(1-x^{2}\right)\right)+O\left(\mu^{2}\right) .
\end{aligned}
$$

Except for the isolated set of points $(x, y)=( \pm 1,0),(63)$ and (64) do not obey the condition (62) for any $\zeta$. It is easily found that no limit cycle solution (i.e., $\mu>0$ ) of (60) and (61) will contain these points. Note that, for the Hamiltonian oscillator $(\mu=0)$, we have an orthogonal decomposition with $\zeta=0$, but as soon as we introduce dissipation, the coordinate basis becomes nonorthogonal, which, in turn, implies AM-PM noise conversion, as discussed in the previous section.

\section{Demir's Nonhyperbolic Oscillator}

In [11], Demir investigates the planar ODE

$$
\begin{aligned}
& \dot{r}=(1-r)^{3} \\
& \dot{\phi}=r
\end{aligned}
$$

which describes a stable nonhyperbolic periodic solution $(r, \phi)=\left(1, t+\phi_{0}\right)$. As was noted in [11], (65) and (66) have both amplitude and frequency that are asymptotically stable; however, no such stability condition exists for the phase.

We can proceed in the same manner as for the system in (45) and (46) which produces the result $f(r)=1 /(1-r)+C$, where, again, $C$ is an integration constant. It is seen that the function $f$ has a singularity at $r=1$, implying that there does not exist an invariant transverse bundle for the oscillator in (65)

\footnotetext{
${ }^{13}$ The $i$ th component of the Lie bracket is calculated as $w_{i}=\left[f, f^{\perp}\right]_{i}=$ $\sum_{j=1}^{2}\left(f_{j}\left(\partial f_{i}^{\perp}\right) /\left(\partial x_{j}\right)-f_{j}^{\perp}\left(\partial f_{i}\right) /\left(\partial x_{j}\right)\right)$, with $\left(x_{1}, x_{2}\right)=(x, y)$
}

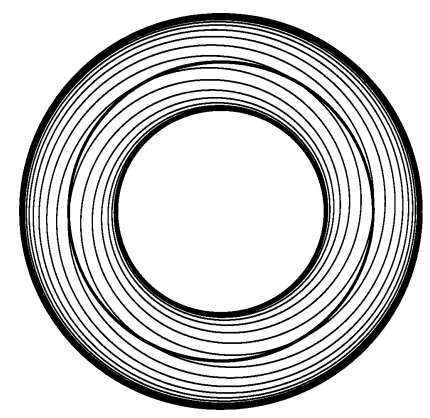

Fig. 5. Periodic solution and isochrone foliation for the perturbed system $\dot{r}=$ $(1-r)^{3}-\beta(1-r), \dot{\phi}=r$ for $\beta=0.1$ (see (65) and (66)). The system contains three limit cycles at $r=(1-\sqrt{\beta}$ [stable], 1 [unstable], $1+\sqrt{\beta}$ [stable] $)=$ $(0.683,1,1.316)$. The isochrones exist in the annulus between the two stable limit cycles; the rest of the plane is a so-called phaseless set [12]. Using the method described in Section VI-A, we find the following expression for the isochrone foliation in this region: $\mathbb{\square}(\phi, r)=\phi-1 /(2 \sqrt{\beta})(\ln (\sqrt{\beta}+r-1)-$ $\ln (\sqrt{\beta}-r+1))$, which is plotted above for $\rrbracket=0, \pi / 6, \pi / 3, \pi, 2 \pi / 3,5 \pi / 6$.

and (66). This, in turn, implies that there cannot exist a linear response representation for this oscillator. This was also discussed in [11], where the author investigates the Floquet response and notes that it does not give the expected result.

In order for a transverse bundle to exist, a manifold has to be normally hyperbolic. However, from (65) and (66), it is clear that, as the orbit nears the periodic solution, the linear response vector field approaches a pure phase (azimuthal) component. Note that it is this lack of normal hyperbolicity, and not the nonhyperbolic amplitude (65), that accounts for the absence of a transverse bundle; indeed, changing (66) to, e.g., $\dot{\phi}=1+(1-r)^{4}$ would produce an isochronous oscillator. Of course, if (65) was hyperbolic, an invariant foliation would be guaranteed to exist.

This is shown in Fig. 5, where we add a small perturbative term to (65), making it hyperbolic. In this scenario, the nonhyperbolic solution bifurcates, producing one unstable and two stable limit cycles. One can gain insight into the phaseless nature of (65) and (66) by noting that the extent of the annulus in Fig. 5, where the isochrone foliation exists, is on the order of $\sqrt{\beta}$; this region hence reduces to the periodic set as $\beta \rightarrow 0$.

\section{ASYMPTOTIC OSCILLATOR PHASE StATISTICS}

As explained in [1], using the decomposition of the linear response flow in (39), we can isolate the phase dynamics of the noise-perturbed circuit as follows: ${ }^{14}$

$$
\frac{\mathrm{d} \alpha}{\mathrm{d} t}=\rho(t+\alpha(t)) \xi(t)
$$

where $\alpha(t)$ is a time variable related to the oscillator phase $\phi$ as $\phi(t)=\left(2 \pi / T_{0}\right) \alpha(t), \rho: \mathbb{R} \rightarrow \mathbb{R}$ is the $T_{0}$ periodic scalar noise modulation function, and $\xi(t): \mathbb{R} \rightarrow \mathbb{R}$ is a unit-variance zero-mean white macro noise source.

${ }^{14}$ Here, we define $\rho^{2}(s)=v^{T}(s) v(s)$, where $s=t+\alpha(t)$ and $v(s)=$ $v_{1}^{T}(s) B\left(x_{s}(s)\right)$, with $B: \mathbb{R}^{n} \rightarrow \mathbb{R}^{n \times p}$ being the noise modulation matrix [1]. While (67) is not identical to the phase SDE used in [1] (see [1, eq. (12)]), the ensemble averages of the noise forcing (right-hand sides) are identical, and the two expressions lead to identical Fokker-Planck formulations. This is seen by inserting the noise modulation function in (67) into (72) and (73) which will produce the drift and diffusion constants used in [1] (see [1, eq. (21)]). Thus, although the individual realizations of the two processes are different, in a statistical sense, they are interchangeable. 
The statistics of the stochastic process described through (67) are characterized by a time-varying probability distribution function, conditioned on deterministic (sharp) phase at time $t_{0}, p\left(x, t \mid x_{0}, t_{0}\right)=\left.\langle\delta(\alpha(t)-x)\rangle\right|_{\alpha\left(t_{0}\right)=x_{0}}$.

Since the phase dynamics are neutrally stable (see the discussion in Section II), the only possible stationary distribution is given by 15

$$
\lim _{t \rightarrow \infty} p\left(x, t \mid x_{0}, t_{0}\right)=1 / T_{0}, x \in\left[-T_{0} / 2 ; T_{0} / 2\right]
$$

where this uniform distribution tells us that the oscillator phase becomes completely random asymptotically with time.

We now introduce the stochastic variable $\beta_{i k}(t, \tau)$ through

$$
\beta_{i k}(t, \tau)=i \alpha(t+\tau)-k \alpha(t) .
$$

As shown in [1], once the asymptotic statistics of (69) are specified for all $i, k$, the phase noise characterization is complete $^{16}$.

We first consider the case $i \neq k$ in (69). Since the asymptotic statistics of both terms are specified by (68), it follows that the expression in (69), for $i \neq k$, will include a completely random term. It then follows that $\beta_{i k}(t, \tau)$ itself must be random for $i \neq k$ and hence will have no effect on the asymptotic phase statistics.

For $i=k$, the variable in (69) will not be random since the randomness is removed in the symmetric difference. The oscillator self-referenced phase (SR-P) $\Delta_{\alpha}(t, \tau)$ is now given as

$$
\beta_{i i}(t, \tau)=i(\alpha(t)-\alpha(t+\tau))=i \Delta_{\alpha}(t, \tau) .
$$

With the aim of characterizing the oscillator SR-P, we now integrate (67) from $t$ to $t+\tau$

$$
\alpha(t+\tau)-\alpha(t)=\int_{t}^{t+\tau} \rho(\eta+\alpha(\eta)) \mathrm{d} w(\eta)
$$

where the Wiener process $w(t): \mathbb{R} \rightarrow \mathbb{R}$ is defined as the integration of the white noise source $\xi(t)$ (i.e., $\mathrm{d} w(t)=\xi(t) \mathrm{d} t)$. The integral on the right-hand side in (71) is stochastic and can hence be specified only through its moments. According to the Stratonovich interpretation [18] of this stochastic integral, the process is characterized through a drift and diffusion coefficient, which is given as

$$
\begin{aligned}
D_{\alpha}(t+x) & \left.\equiv \lim _{\tau \rightarrow 0} \frac{1}{\tau}\langle(\alpha(t+\tau)-\alpha(t))\rangle\right|_{\alpha(t)=x} \\
& =\rho(t+x) \frac{\partial \rho(t+x)}{\partial x} \\
D_{\alpha \alpha}(t+x) & \left.\equiv \lim _{\tau \rightarrow 0} \frac{1}{\tau}\left\langle(\alpha(t+\tau)-\alpha(t))^{2}\right\rangle\right|_{\alpha(t)=x} \\
& =\rho^{2}(t+x) .
\end{aligned}
$$

${ }^{15}$ This can also be written as $\left.\lim _{t \rightarrow \infty} p\left(x, t \mid x_{0}, t_{0}\right)=0, x \in\right]-\infty ; \infty[$ if we interpret $\alpha$ on the real line since a completely random stochastic variable on an unbounded sample space must have zero probability distribution everywhere. See also [1, Theorem 7.1] for a mathematical proof.

${ }^{16}$ The oscillator autocorrelation function is given as $R(t, \tau)=E\left[x_{s}(t+\right.$ $\left.\alpha(t)) x_{s}^{*}(t+\tau+\alpha(t+\tau))\right]=\sum_{i=-\infty}^{\infty} \sum_{k=-\infty}^{\infty} X_{i} X_{k}^{*} \exp \left(j \omega_{0}(i-\right.$ $k) t) \exp \left(-j \omega_{0} k \tau\right) E\left[\exp \left(-j \omega_{0} \beta_{i k}(t, \tau)\right)\right]$, where $X_{k}$ is the $k$ th Fourier component vector and $\omega_{0}=2 \pi / T_{0}$. The oscillator phase noise spectrum is then found by Fourier transforming this expression [1].

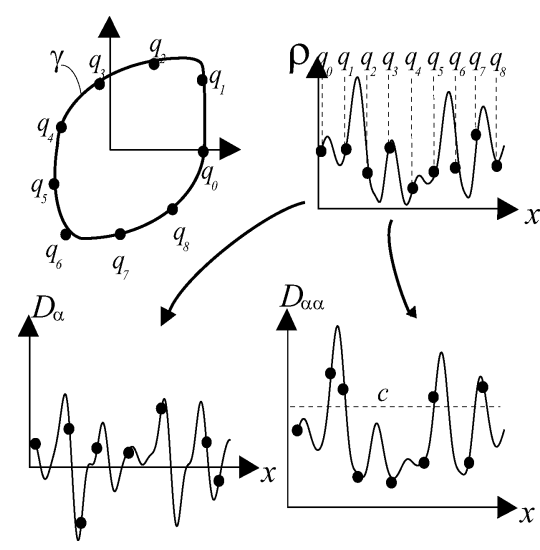

Fig. 6. In the limit $t \rightarrow \infty$, the oscillator phase ensemble is completely diffused, corresponding to a uniform distribution on the limit cycle $\gamma$. This is illustrated in the top-left part of the figure, where the limit cycle is discretized into $M=9$ time points $\left\{q_{j}\right\}$, with the oscillator ensemble being uniformly distributed to each of the $M$ points. The periodic forcing function $\rho$, as introduced in (67), is shown in the top-right part of the figure. The effective drift coefficient is then derived as (see the lower left figure) $\lim _{t \rightarrow \infty} \mathrm{d} E\left[\Delta_{\alpha}(t, \tau)\right] / \mathrm{d} \tau=\lim _{M \rightarrow \infty} \sum_{j-0}^{M-1} D_{\alpha}\left(t+q_{j}\right) / M=0$, and the effective diffusion coefficient becomes (see the lower right figure) $\lim _{t \rightarrow \infty} \mathrm{d} V\left[\Delta_{\alpha}(t, \tau)\right] / \mathrm{d} \tau=\lim _{M \rightarrow \infty} \sum_{j=0}^{M-1} D_{\alpha \alpha}\left(t+q_{j}\right) / M=c$.

The functions in (72) and (73) specify the time evolution of the SR-P mean and second moment, respectively, conditioned on the phase taking on the deterministic (sharp) value $x$ at time $t$. With regard to capturing the asymptotic statistics of $\Delta_{\alpha}(t, \tau)$, the obvious problem with (72) and (73) is then that $\lim _{t \rightarrow \infty} \alpha(t)$ is not sharp but instead completely random, as specified by (68). However, since we know the stationary distribution of the variable $\alpha$, as given by (68), we can derive the ensemble-averaged evolution constants, which are referred to here as the effective drift constants

$$
\lim _{t \rightarrow \infty} \frac{\mathrm{d} E\left[\Delta_{\alpha}(t, \tau)\right]}{\mathrm{d} \tau}=\frac{1}{T_{0}} \int_{0}^{T_{0}} D_{\alpha}(t+x) \mathrm{d} x=0
$$

and the effective effective diffusion constant ${ }^{17}$

$$
\lim _{t \rightarrow \infty} \frac{\mathrm{d} V\left[\Delta_{\alpha}(t, \tau)\right]}{\mathrm{d} \tau}=\frac{1}{T_{0}} \int_{0}^{T_{0}} D_{\alpha \alpha}(t+x) \mathrm{d} x=c
$$

where it was indicated that (72), a periodic function multiplied by its own derivative, has zero dc, while (73) must have a dc component that we, following [1], denote with $c$. The previous calculations are illustrated for a discrete phase ensemble in Fig. 6.

The aforementioned calculations could be redone for $\tau<0$, and one would refind (74), while (75) becomes

$$
\lim _{t \rightarrow \infty} \frac{\mathrm{d} V\left[\Delta_{\alpha}(t,-\tau)\right]}{\mathrm{d} \tau}=-c .
$$

By integrating (74), (75), and (76) with the initial conditions $\lim _{t \rightarrow \infty} E\left[\Delta_{\alpha}(t, 0)\right]=0$ and $\lim _{t \rightarrow \infty} V\left[\Delta_{\alpha}(t, 0)\right]=0$, we find the asymptotic SR-P mean and power as

$$
\begin{aligned}
& \lim _{t \rightarrow \infty} E\left[\Delta_{\alpha}(t, \tau)\right]=0 \\
& \lim _{t \rightarrow \infty} V\left[\Delta_{\alpha}(t, \tau)\right]=c|\tau|
\end{aligned}
$$

${ }^{17}$ Here, we use (74) to set $V[X]=E\left[X^{2}\right]-(E[X])^{2}=E\left[X^{2}\right]$. 
and since $\lim _{t \rightarrow \infty} \Delta_{\alpha}(t, \tau)$ is a Gaussian stochastic variable, which follows from the Gaussian nature of the noise, we find the following asymptotic distribution:

$$
\begin{aligned}
\lim _{t \rightarrow \infty} p\left(\Delta_{\alpha}(t, \tau)\right) & =\lim _{t \rightarrow \infty}\left\langle\delta\left(\Delta_{\alpha}(t, \tau)-x\right)\right\rangle \\
& =\frac{1}{\sqrt{4 \pi c|\tau|}} \exp \left(-\frac{x^{2}}{c|\tau|}\right)
\end{aligned}
$$

which is seen to be identical to what was found in [1], as was, of course, to be expected. However, the aforesaid derivation constitutes a serious reduction in the amount and complexity of the calculations needed to reach (79). Equation (79) completes the asymptotic characterization of the variable $\beta_{i k}(t, \tau)$ and, hence, of the phase noise scenario (see Footnote 16).

\section{CONCLUSION AND Future WORK}

This paper has documented the construction of the oscillator linear response map, with the derivation being based on the simple geometrical ideas implied from the assumption of a hyperbolic solution. This general model was shown to naturally include the well-known phase macromodel as a special case. A simplified derivation of the asymptotic oscillator phase statistics was included. We intend to conduct a more thorough investigation of the topics discussed in Section VI-C.

\section{REFERENCES}

[1] A. Demir, A. Mehrotra, and J. Roychowdhury, "Phase noise in oscillators: A unifying theory and numerical methods for characterization," IEEE Trans. Circuits Syst. I, Fundam. Theory Appl., vol. 47, no. 5, pp. 655-674, May 2000.

[2] A. Demir, "Phase noise and timing jitter in oscillators with colorednoise sources," IEEE Trans. Circuits Syst. I, Fundam. Theory Appl., vol. 49, no. 12, pp. 1782-1791, Dec. 2002.

[3] A. Demir, "Computing timing jitter from phase noise spectra for oscillators and phase-locked loops with white and $1 / f$ noise," IEEE Trans. Circuits Syst. I, Reg. Papers, vol. 53, no. 9, pp. 1869-1884, Sep. 2006.

[4] F. X. Kaertner, "Determination of the correlation spectrum of oscillators with low noise," IEEE Trans. Microw. Theory Tech., vol. 37, no. 1, pp. 90-101, Jan. 1989.

[5] F. X. Kaertner, "Analysis of white and $f^{-\alpha}$ noise in oscillators," Int. J. Circuit Theory Appl., vol. 18, no. 5, pp. 485-519, Sep. 1990.

[6] T. H. Lee and A. Hajimiri, "Oscillator phase noise: A tutorial," IEEE J. Solid-State Circuits, vol. 35, no. 3, pp. 326-336, Mar. 2000.

[7] K. Kurokawa, "Noise in synchronized oscillators," IEEE Trans. Microw. Theory Tech., vol. MTT-16, no. 4, pp. 234-240, Apr. 1968.

[8] G. J. Coram, "A simple 2-D oscillator to determine the correct decomposition of perturbations into amplitude and phase noise," IEEE Trans. Circuits Syst. I, Fundam. Theory Appl., vol. 48, no. 7, pp. 896-898, Jul. 2001.

[9] T. Djurhuus, V. Krozer, J. Vidkjaer, and T. Johansen, "Nonlinear analysis of a cross-coupled quadrature harmonic oscillator," IEEE Trans. Circuits Syst. I, Fundam. Theory Appl., vol. 52, no. 11, pp. 2276-2285, Nov. 2005.

[10] T. Djurhuus, V. Krozer, J. Vidkjær, and T. Johansen, “AM to PM noise conversion in a cross-coupled quadrature harmonic oscillator," Int. J. RF Microw. Comput.-Aided Eng., vol. 16, no. 1, pp. 34-41, Jan. 2005.

[11] A. Demir, "Fully nonlinear oscillator noise analysis: An oscillator with no asymptotic phase," Int. J. Circuit Theory Appl., vol. 35, no. 2, pp. 175-203, Mar. 2007.

[12] J. Guckenheimer, "Isochrons and phaseless sets," J. Math. Biol., vol. 1, no. 3, pp. 259-273, Sep. 1975.

[13] A. T. Winfree, The Geometry of Biological Time, 2nd ed. New York: Springer-Verlag, 2000.

[14] F. C. Hoppensteadt and E. M. Izhikevich, Weakly Connected Neural Networks. New York: Springer-Verlag, 1997.
[15] C. Chicone, Ordinary Differential Equations with Applications. New York: Springer-Verlag, 2006.

[16] C. Chicone and W. Liu, "Asymptotic phase revisited," J. Differ. Equ., vol. 204 , no. 1 , pp. 227-246, Sep. 2004.

[17] A. B. Carlson, Communication Systems, 3rd ed. New York: McGrawHill, 1986.

[18] H. Risken, Fokker-Planck Equation-Methods of Solution and Applications. New York: Springer-Verlag, 1989.

[19] D. Leeson, "A simple model of feedback oscillator noise," Proc. IEEE, vol. 54, no. 2, pp. 429-430, Feb. 1966.

[20] B. Razavi, "The study of phase noise in CMOS oscillators," IEEE J. Solid-State Circuits, vol. 31, no. 3, pp. 331-343, Mar. 1996.

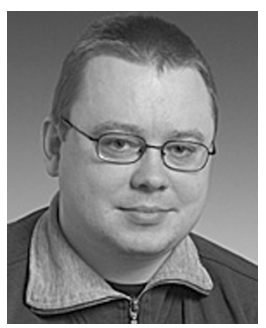

Torsten Djurhuus received the M.S. and Ph.D. degrees in electrical engineering from the Technical University of Denmark, Lyngby, Denmark, in 2003 and 2007 , respectively.

He is currently a Research Assistant with the DTU Electrical Engineering Department. His research interests include nonlinear circuit analysis, circuit noise analysis, monolithic microwave integrated circuit design, and RF oscillator design.

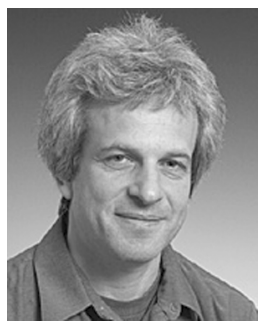

Viktor Krozer (M'91-SM'03) received the Dipl.-Ing. and Dr.-Ing. degrees in electrical engineering from the Technical University of Darmstadt (TU Darmstadt), Darmstadt, Germany, in 1984 and 1991, respectively.

In 1991, he became a Senior Scientist with TU Darmstadt, where he was involved in activities concerning high-temperature microwave devices and circuits and submillimeter-wave electronics. From 1996 to 2002, he was a Professor with the Technical University of Chemnitz, Chemnitz, Germany. Since 2002, he has been a Professor with the ElectroScience Section, DTU Electrical Engineering Department, Technical University of Denmark, Lyngby, Denmark, where he heads the Microwave Technology Group. His research areas include terahertz electronics, monolithic microwave integrated circuits, nonlinear circuit analysis and design, device modeling, and remote sensing instrumentation.

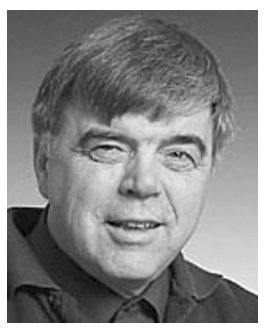

Jens Vidkjær (S'72-M'72) received the M.S. and Ph.D. degrees from the Technical University of Denmark, Lyngby, Denmark, in 1968 and 1975, respectively.

Since 1970, he has been with the Electronics Laboratory and the Semiconductor Laboratory, Technical University of Denmark, where he is currently a Reader with the DTU Electrical Engineering Department. His research areas include RF-power amplifier design, computer-aided-design methods, device modeling, measurement accuracies, and monolithic microwave integrated circuit design.

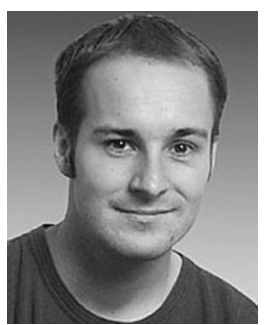

Tom K. Johansen (S'02-M'04) received the M.S. and $\mathrm{Ph} . \mathrm{D}$. degrees in electrical engineering from the Technical University of Denmark, Lyngby, Denmark, in 1999 and 2003, respectively.

In 1999, he joined the Electromagnetic Systems Section, DTU Electrical Engineering Department, Technical University of Denmark, where he is currently an Associate Professor. From September 2001 to March 2002, he was a Visiting Scholar with the Center for Wireless Communication, University of San Diego, San Diego, CA. His research areas include the modeling of HBT devices, nonlinear circuit analysis, millimeter-wave integrated circuit design, and electromagnetic simulation. 